\title{
Comportamiento clínico de los tumores cardíacos desde el feto hasta el adulto: serie multicéntrica de 38 pacientes
}

\author{
Pilar Arnaiz G ${ }^{\mathbf{1} 2}$, Isabel Toledo G ${ }^{\mathbf{1}, 2}$, Arturo Borzutzky S $\mathbf{1}$, \\ G onzalo U rcelay $M^{1,2}$, Felipe Heusser $R^{1,2}$, \\ Francisco $G$ aray $\mathrm{G}^{1,2}$, Elisa Castillo $\mathrm{N}^{1,3}$, Lida Toro $\mathrm{R}^{3}$, \\ Pedro Becker $\mathbf{R}^{2}$, Claudio Arretz $\mathbf{V}^{2}$, Samuel Córdova $\mathbf{A}^{2}$. \\ Cardiac tumors in children and \\ adults: A retrospective study
}

Background: Cardiac tumors are very uncommon at all ages. There are important clinical differences between children and adults in the behavior of these tumors. Aim: To compare the behavior of primary and secondary cardiac tumors, from fetal age to adults. Patients and Method: Multicentric retrospective analysis of 38 children and adults with cardiac tumors, evaluated with echocardiography between January 1995 and August 2001. Medical records, echocardiographic and radiological examinations, surgical protocols and pathologic examinations were reviewed. Follow-up was obtained through data on medical records or calling patients by telephone. Results: Tumors were diagnosed in 38 patients ( 13 children and 25 adults), from a total of 31.800 echocardiograms. In children the diagnosis was made by fetal, transthoracic or transesophageal echocardiography in $23.6 \%$ and $8 \%$ of cases, respectively. Eighty five percent were primary (10 benign and 1 malignant) and $15 \%$, secondary tumors. Fifty four percent were rhabdomyomas and $75 \%$ regressed spontaneously. Seventy seven percent were symptomatic and $31 \%$ were treated with surgery. During a follow up of $44 \pm 35$ months, $31 \%$ of patients died. In adults, $76 \%$ of tumors were diagnosed by transthoracic and $20 \%$ by transesophageal echocardiography. Seventy six percent were primary (18 benign and 1 malignant) and 24\% secondary tumors. Fifty six percent were myxomas. Ninety two percent were symptomatic and $84 \%$ were treated surgically. Twenty percent of patients died in the early postoperative period. No adult patients had a follow-up. Conclusions: Rhabdomyomas were solely found in children. In adults, myxomas are the predominat cardiac tumors. Primary and metastasic malignant tumors are observed both in children and in adults (Rev Méd Chile 2006; 134: 1135-45).

(Key w ords: Echocardiography; Heart neoplasms; Myxoma; Rhabdomyoma).

Recibido el 19 de julio, 2005. Aceptado el 14 de marzo, 2006.

${ }^{1}$ Departamento de Pediatría y ${ }^{2}$ Departamento de Enfermedades Cardiovasculares, Facultad de Medicina, Pontificia Universidad Católica de Chile. ${ }^{3}$ Servicio de Pediatría, Hospital Dr. Sótero del Río, Servicio de Salud Metropolitano Sur-Oriente.

Correspondencia a: Dra. Pilar Arnaiz G. Lira 85, 50 Piso, Santiago. Teléfono: 3543766. Fax: 6384307. E mail: parnaiz@med.puc.cl 
Tos tumores cardíacos primarios constituyen ـuna patología muy poco frecuente a toda edad, pero por la condición del órgano que comprometen, tienen una especial relevancia clínica y un pronóstico poco predecible. La más reciente revisión de 12.485 autopsias mostró una incidencia de $0,02 \%$ (doscientos tumores por cada millón de autopsias) ${ }^{1}$. La presentación varía dependiendo de la edad, tipo de tumor y ubicación, existiendo tumores del corazón desde el feto hasta el adulto mayor ${ }^{2-5}$. La incidencia de tumores cardíacos ha ido en aumento en las últimas décadas, asociado a los avances en imagenología del corazón, especialmente la ecocardiografía, como herramienta diagnóstica fundamental ${ }^{2,6}$. Los tumores primarios benignos son más frecuentes que los malignos, no obstante, los tumores benignos pueden evolucionar muy desfavorablemente, por los múltiples efectos hemodinámicos que pueden presentar ${ }^{7-14}$. Los tumores secundarios son 20 veces más frecuentes que los tumores primarios, pero muchas veces éstos no se diagnostican ni tratan, debido a que se presentan en pacientes con cáncer muy avanzado $2,34,36$.

El objetivo de este estudio retrospectivo fue comparar el comportamiento de los tumores cardíacos primarios y secundarios, desde la edad fetal hasta el adulto. Se incluyó la casuística de dos instituciones y en un período de seis años.

\section{PACIENTES Y MÉTODO}

Se realizó una revisión de 38 pacientes con diagnóstico de tumor cardíaco, evaluados mediante ecocardiografía en el Hospital Clínico de la Pontificia Universidad Católica de Chile y en el Hospital Dr. Sótero del Río, entre enero de 1995 y agosto de 2001. Se incluyeron pacientes con diagnóstico de tumores cardíacos primarios y secundarios en edades fetal, infantil y adulta. Durante este período, se realizaron en dichos centros 2.300 ecocardiografías fetales, 14.000 ecocardiografías en niños y adolescentes y 15.500 ecocardiografías en adultos.

Se obtuvo información de cada paciente mediante la revisión de historias clínicas, exámenes ecocardiográficos y radiológicos, protocolos operatorios, estudios anatomopatológicos y de seguimiento, según registros de fichas clínicas y contacto telefónico. El tipo de tumor fue identificado mediante estudio anatomopatológico posoperatorio en aquellos casos en que se sometió al paciente a intervención quirúrgica, o por comparación de la apariencia ecocardiográfica, radiológica o ambos, con la descripción de tumores en la literatura. El tiempo promedio de seguimiento en los tumores infantiles fue de $44 \pm 35$ meses con un rango entre 9 días y 60 meses. No se realizó seguimiento posterior al alta hospitalaria en los pacientes adultos.

Se analizaron los datos con el programa SPSS 10.0. Los valores se expresan en términos de promedio \pm desviación estándar.

\section{RESUlTADos}

En total, se analizaron 38 pacientes con diagnóstico de tumor cardíaco. De éstos, 13 eran niños y 25 adultos. De los niños, 3 fueron diagnosticados en la etapa fetal. La incidencia de tumores cardíacos en el total de ecocardiografías efectuadas durante el período del estudio, expresada en tasa por 1.000 ecos fue de: 1,3 tumores fetales, 0,7 tumores infantiles y 1,6 tumores adultos.

Se analizó por separado la presentación clínica de las series infantil y adulta y, posteriormente, se efectuó una comparación entre ambas.

Serie infantil. En el grupo de 13 pacientes pediátricos (Tabla 1) la edad promedio de presentación, excluyendo los casos de diagnóstico prenatal, fue de 61 meses, con un rango amplio entre 4 días y 12 años. Sólo cuatro pacientes fueron diagnosticados antes del año de vida, incluyendo los tres pacientes con diagnóstico prenatal. El 53,8\% de los pacientes fueron de sexo masculino.

Tres tumores fueron diagnosticados mediante ecocardiografía fetal (23\%), ocho por ecocardiografía transtorácica (ECOTT) (61\%), uno por ecocardiografía transesofágica (ECOTE) (8\%) y uno mediante resonancia magnética cardíaca como método complementario (8\%).

Once pacientes (85\%) presentaron tumores primarios del corazón, diez eran benignos y uno sólo era maligno (rabdomiosarcoma cardíaco). En el grupo de tumores benignos, siete fueron rabdomiomas, un fibroma, un mixoma y un tumor de células inflamatorias. Dos pacientes presentaron 
Tabla 1. C aracterísticas de pacientes y tumores de la serie infantil

\begin{tabular}{|c|c|c|c|c|c|c|c|}
\hline $\begin{array}{l}\text { № } \\
\text { caso }\end{array}$ & $\begin{array}{c}\text { Edad } \\
\text { diagnóstico }\end{array}$ & Sexo & $\begin{array}{l}\text { Método } \\
\text { diagnóstico }\end{array}$ & Tipo tumoral & $\begin{array}{c}\text { № } \\
\text { lesiones }\end{array}$ & Localización & $\begin{array}{l}\text { Presentación } \\
\text { clínica }\end{array}$ \\
\hline 1 & 4,5 á & F & EcoTT & Fibroma & 1 & VD & Asintomático \\
\hline 2 & 4,1 á & M & EcoTT & Rabdomioma & 3 & VI & Asintomático \\
\hline 3 & Fetal & $\mathrm{F}$ & Eco F & Rabdomioma & 1 & VI (M. papilar) & Convulsiones \\
\hline 4 & Fetal & M & Eco F & Rabdomioma & 3 & $\mathrm{VD}, \mathrm{VI}$ & Asintomático \\
\hline 5 & Fetal & F & Eco F & Rabdomioma & Múltiple & $\begin{array}{l}\text { Septum IV, } \\
\text { VD, AD }\end{array}$ & $\begin{array}{l}\text { IC, TPSV por } \\
\text { WPW }\end{array}$ \\
\hline 6 & 2 á & M & EcoTT & Mixoma & 1 & $\begin{array}{l}\text { Septum primum AI, } \\
\text { protruyendo VI }\end{array}$ & $\begin{array}{c}\text { Síncope } \\
\text { Edema pulmonar }\end{array}$ \\
\hline 7 & 4,7 á & M & EcoTT, RM & Rabdomiosarcoma & 1 & $\mathrm{AD}$ & $\begin{array}{c}\text { Derrame } \\
\text { pericárdico }\end{array}$ \\
\hline 8 & 3,7á & M & EcoTT & Rabdomioma & 1 & $A D$, surco $A V$ & Convulsiones \\
\hline 9 & 9,1 á & $\mathrm{F}$ & EcoTT & $\begin{array}{l}\text { Metástasis rabdomio- } \\
\text { sarcoma hepático }\end{array}$ & 1 & $\mathrm{AI}$ & Embolia cerebral \\
\hline 10 & 12,2 á & M & EcoTE & $\begin{array}{c}\text { Tumor de células } \\
\text { Inflamatorias } \\
\text { (pseudotumor) }\end{array}$ & 1 & $\begin{array}{c}\mathrm{AD} \text {, anillo } \\
\text { tricuspídeo } \\
\text { protruyendo VD }\end{array}$ & $\begin{array}{l}\text { Síndrome febril } \\
\text { prolongado }\end{array}$ \\
\hline 11 & 1,4 á & $\mathrm{F}$ & EcoTT & Rabdomioma & 1 & septum VI & TPSV por WPW \\
\hline 12 & 9 á & $\mathrm{F}$ & EcoTT & Metástasis hepatoma & 1 & $\mathrm{AD}$ & Síndrome CI \\
\hline 13 & $4 \mathrm{~d}$ & M & EcoTT & Rabdomioma & 1 & VD anillo tricúspideo & Trisomía 18, CIV \\
\hline
\end{tabular}

á: años; d: días; M: masculino; F: femenino; EcoTT: ecocardiografía transtorácica; EcoTE: ecocardiografía transesofágica; Eco F: ecocardiografía fetal; RM: resonancia magnética; VD: ventrículo derecho; VI: ventrículo izquierdo; AD: aurícula derecha; IV: interventricular; AI: aurícula izquierda; AV: atrioventricular; TPSV: taquicardia paroxística supraventricular; WPW: Wolf Parkinson White; CIV: comunicación interventricular; IC: Insuficiencia Cardíaca; CI: cava inferior.

tumores secundarios consistentes en metástasis cardíacas de tumores previamente diagnosticados.

Diez tumores (77\%) eran únicos y tres múltiples, los tres rabdomiomas.

Respecto a la localización de los tumores, en cuatro pacientes comprometían la aurícula derecha (AD), en dos, la aurícula izquierda (AI), en tres, el ventrículo izquierdo (VI) y en dos, el ventrículo derecho (VD). En dos casos, ambos rabdomiomas, el compromiso era de más de una cavidad, con uno en ambas cavidades derechas y en el otro en ambos ventrículos.

Presentación clínica. De los 13 pacientes, sólo tres fueron asintomáticos al momento del diagnóstico, dos con rabdomioma y uno con fibroma. De los rabdomiomas asintomáticos, uno tenía diagnóstico prenatal del tumor y nació asintomático, mientras el segundo, se diagnosticó en una ecocardiografía efectuada por soplo de carácter fun- cional. A raíz del hallazgo del tumor cardíaco, se estudió y resultó tener además una esclerosis tuberosa.

Los pacientes sintomáticos fueron 10: un niño con síndrome febril prolongado, dos pacientes con arritmias por taquicardia supraventricular por Wolf Parkinson White (WPW), (una de las cuales presentó además insuficiencia cardíaca por obstrucción al flujo intracardiaco por rabdomioma múltiple), un niño con síntomas de presíncope y edema pulmonar agudo; dos pacientes con convulsiones y retardo del desarrollo psicomotor, un niño con signos de trisomía 18 y comunicación interventricular (CIV), un niño con derrame pericárdico recurrente por infiltración pericárdica, una niña con embolia cerebral por metástasis en la AI y una niña con síndrome de cava inferior.

Tratamiento y seguimiento. Un paciente portador de rabdomioma no siguió en control. Cuatro 
pacientes fueron sometidos a intervención quirúrgica: un mixoma, un tumor de células inflamatorias, un rabdomiosarcoma y una metástasis de hepatoma, efectuándose resección total o parcial del tumor cardíaco, reparación de cavidades y válvulas comprometidas. En el caso del rabdomiosarcoma, se efectuó además una ventana pericárdica. Este último paciente fue sometido a trasplante cardíaco debido a una miocardiopatía restrictiva, secundaria a radioterapia. En los ocho pacientes no operados, seis rabdomiomas, un fibroma y la niña con rabdomiosarcoma hepático, que se consideró fuera del alcance quirúrgico, se indicó seguimiento clínico-ecográfico, que se concretó en cinco de ellos.

Durante el tiempo de seguimiento de este estudio (8 años), tres pacientes fallecieron tempranamente: el paciente con trisomía 18 y rabdomioma de VD falleció a los 15 días de vida por su enfermedad de base, una paciente con rabdomioma múltiple y taquicardia supraventricular por WPW falleció a los 2 meses de vida con insuficiencia cardíaca y la tercera paciente falleció a los 10 años de vida, a consecuencia del rabdomiosarcoma hepático y las múltiples metástasis.

De los nueve pacientes actualmente en control, todos están asintomáticos del punto de vista cardiovascular. Durante el seguimiento han recibido distintas terapias. El caso de la niña con un fibroma de VD, tiempo después del diagnóstico comenzó a presentar extrasistolía ventricular frecuente y tuvo un episodio de presíncope. Inició tratamiento con atenolol y continuó seguimiento con Holter de arritmias, sin volver a presentar síntomas. Una de las pacientes con rabdomioma y taquicardia supraventricular por WPW recibe amiodarona y está asintomática. En el niño con mixoma de AI, un año después de la extirpación del tumor, recidivó y hubo que reoperarlo. Actualmente está asintomático y libre de tumor. El adolescente con el tumor de células inflamatorias no ha recidivado y también continúa asintomático. En el caso del niño con rabdomiosarcoma primario, actualmente está cursando su segundo año postrasplante cardíaco en buenas condiciones cardiovasculares. La niña con hepatoma y metástasis resecada de $\mathrm{AD}$ continúa asintomática del punto de vista cardiovascular. Los tres pacientes restantes tenían rabdomiomas y continúan asintomáticos cardiovasculares.
De los cuatro pacientes con rabdomioma en control, se observó regresión espontánea parcial en tres de ellos y en uno ha permanecido igual durante 5 años de seguimiento.

Serie adulta. En los 25 pacientes adultos (Tabla 2), la edad de presentación promedio fue de $55 \pm 16,8$ años, con un rango entre 19 y 82 años. El 64\% de los pacientes era de sexo femenino.

Los tumores fueron diagnosticados mediante ECOTT en 19 casos (76\%), ECOTE en 5 casos (20\%), uno de ellos como método único de diagnóstico y 4 como complemento a la ECOTT y en un caso fue la angiografía la herramienta diagnóstica.

En 19 pacientes (76\%) los tumores cardíacos eran primarios, 18 eran benignos y uno maligno (leiomiosarcoma cardíaco). De los tumores benignos el tipo tumoral predominante fue el mixoma, presente en 14 de ellos. Tres pacientes se presentaron con fibroelastoma y un paciente con un hamartoma. En 6 pacientes (24\%) el tumor era secundario, constituido por una metástasis cardíaca proveniente de otro sitio primario (carcinoma cervicouterino, adenocarcinoma pulmonar, sarcoma retroperitoneal, linfoma, tumor testicular, mieloma).

Los tumores eran masas únicas en todos los casos, excepto en 3: un caso de fibroelastoma de válvula aórtica en que se observaban dos tumores de $1,5 \mathrm{~cm}$ de diámetro, y en dos casos de metástasis cardíacas de mieloma y adenocarcinoma pulmonar.

La localización de los tumores primarios dependió del tipo tumoral: 13 de 14 mixomas se localizaron en AI y 1 en VD. Dos de los fibroelastomas se localizaron en la válvula aórtica y uno en VI. Los dos tumores primarios restantes, hamartoma y leiomiosarcoma, se ubicaron en las cavidades derechas.

Presentación clínica. De los 25 pacientes de la serie, 2 pacientes con mixoma fueron asintomáticos al momento del diagnóstico. Se trató de dos personas jóvenes de 39 y 44 años respectivamente, que dentro de un chequeo general se solicitó un ecocardiograma, el que mostró el tumor.

Los pacientes sintomáticos fueron 23 al momento del diagnóstico, presentándose la mayoría con insuficiencia cardiaca y disnea de diversa consideración, que en algunos casos llegó a insuficiencia cardíaca severa y edema pulmonar 
Tabla 2. C aracterísticas de pacientes y tumores de la serie de adultos

\begin{tabular}{|c|c|c|c|c|c|c|c|}
\hline $\begin{array}{l}\text { № } \\
\text { caso }\end{array}$ & $\begin{array}{c}\text { Edad } \\
\text { diagnóstico }\end{array}$ & Sexo & $\begin{array}{c}\text { Método } \\
\text { diagnóstico }\end{array}$ & Tipo tumoral & $\begin{array}{c}\text { № } \\
\text { lesiones }\end{array}$ & Localización & $\begin{array}{l}\text { Presentación } \\
\text { clínica }\end{array}$ \\
\hline 1 & 36 & $\mathrm{~F}$ & EcoTT & $\begin{array}{c}\text { Metástasis } \\
\text { Ca Cervicouterino }\end{array}$ & 1 & VD & IC \\
\hline 2 & 44 & F & EcoTT & Mixoma & 1 & $\mathrm{AI}$ & IC \\
\hline 3 & 22 & F & EcoTT & $\begin{array}{l}\text { Metástasis sarcoma } \\
\text { retroperitoneal }\end{array}$ & 1 & VCI, AD & Síndrome VCI \\
\hline 4 & 45 & M & EcoTT & Mixoma & 1 & AI & $\mathrm{FA}$ \\
\hline 5 & 82 & F & EcoTT & Mixoma & 1 & $\mathrm{AI}$ & $\mathrm{FA}$ \\
\hline 6 & 73 & $\mathrm{~F}$ & EcoTT & Leiomiosarcoma & 1 & VCS, AD, VD, AP & IC \\
\hline 7 & 49 & M & EcoTT & Mixoma & 1 & $\mathrm{AI}$ & IC \\
\hline 8 & 47 & $\mathrm{~F}$ & EcoTT & Mixoma & 1 & $\mathrm{AI}$ & IC \\
\hline 9 & 74 & F & EcoTT & Mixoma & 1 & $\mathrm{AI}$ & Embolia cerebral \\
\hline 10 & 67 & F & EcoTT & Mixoma & 1 & $\mathrm{AI}, \mathrm{VI}$ & IC \\
\hline 11 & 63 & M & EcoTT & Mixoma & 1 & $\mathrm{AI}$ & IC \\
\hline 12 & 69 & $\mathrm{~F}$ & EcoTT, EcoTE & Mixoma & 1 & AI & Embolia cerebral \\
\hline 13 & 74 & F & EcoTT & Fibroelastoma & 1 & $\begin{array}{l}\text { VI (músculo } \\
\text { papilar) }\end{array}$ & Embolia cerebral \\
\hline 14 & 39 & M & EcoTT, EcoTE & Mixoma & 1 & $\mathrm{AI}$ & Asintomático \\
\hline 15 & 70 & F & EcoTT, EcoTE & Fibroelastoma & 2 & Válvula aórtica & Embolia cerebral \\
\hline 16 & 53 & M & EcoTT, EcoTE & Mixoma & 1 & AI & $\mathrm{FA}$ \\
\hline 17 & 44 & F & EcoTT & Mixoma & 1 & AI & Asintomático \\
\hline 18 & 58 & F & EcoTT & Mixoma & 1 & $\mathrm{AI}$ & Sintomas de CIA \\
\hline 19 & 63 & M & Angio & $\begin{array}{l}\text { Metástasis linfoma } \\
\text { indiferenciado }\end{array}$ & 1 & VCI, AD & Síndrome VCI \\
\hline 20 & 66 & M & EcoTT & Metástasis mieloma & múltiples & VI & IC \\
\hline 21 & 49 & F & EcoTE & Hamartoma & 1 & VCI, AD & Síndrome VCI \\
\hline 22 & 40 & F & EcoTT & $\begin{array}{l}\text { Metástasis Adeno- } \\
\text { carcinoma pulmonar }\end{array}$ & $\begin{array}{l}\text { Infiltración } \\
\text { tumoral }\end{array}$ & $\mathrm{VD}, \mathrm{VI}$ & $\begin{array}{l}\text { Derrame } \\
\text { pericárdico }\end{array}$ \\
\hline 23 & 77 & F & EcoTT & Fibroelastoma & 1 & Válvula aórtica & IC \\
\hline 24 & 19 & M & EcoTT & $\begin{array}{l}\text { Metástasis tumor } \\
\text { testicular }\end{array}$ & $\begin{array}{l}\text { Infiltración } \\
\text { tumoral }\end{array}$ & $\begin{array}{c}\text { VD, } \\
\text { pericardio }\end{array}$ & $\begin{array}{c}\text { Derrame } \\
\text { pericárdico }\end{array}$ \\
\hline 25 & 53 & M & EcoTT & Mixoma & 1 & VD & IC \\
\hline
\end{tabular}

M: masculino; F: femenino; EcoTT: ecocardiografía transtoráxica; EcoTE: ecocardiografía transesofágica; Angio: angiografía; VD: ventrículo derecho; VI: ventrículo izquierdo; AD: auńcula derecha; AI: aunícula izquierda; FA: fibrilación auricular; IC: Insuficiencia Cardiaca; VCS: vena cava superior, VCI: vena cava inferior, AP: arteria pulmonar, CIA: comunicación interauricular

agudo. Cuatro pacientes se presentaron con embolización a distancia y accidentes vasculares encefálicos. Tres pacientes presentaban arritmias, consistente en fibrilación auricular. Dos pacientes presentaron derrame pericárdico importante, ambos tumores malignos secundarios con compromiso pericárdico. Tres pacientes con compromiso de vena cava inferior y aurícula derecha presentaron hipertensión venosa severa. En el caso de un mixoma de AI se observó una comunicación interauricular (CIA) concomitante.

Tratamiento y seguimiento. Sólo en 4 casos no se realizó intervención quirúrgica, ya sea por enfermedad avanzada, contraindicación médica de cirugía cardiaca, o no continuar seguimiento en nuestras instituciones. El tratamiento realizado en los otros 21 casos fue resección tumoral con decorticación cardíaca en un caso. 
Cinco pacientes fallecieron, dos que estaban fuera del alcance quirúrgico y tres en el posoperatorio temprano. En ninguno de los pacientes se realizó seguimiento posterior al alta hospitalaria.

\section{DisCUSIÓN}

Los tumores cardíacos son infrecuentes, y dada su naturaleza esporádica, el tratamiento y pronóstico están basados en la documentación de casos y la analogía de tumores originados en otras ubicaciones.

En esta revisión destacamos importantes diferencias en el comportamiento de los tumores cardíacos según la edad de los pacientes (Tabla $3)$, hecho ya descrito en la literatura.

La mayoría de los tumores cardíacos fueron primarios en adultos, y niños: en la serie infantil correspondieron a $85 \%$ de los casos y para la serie de adultos $76 \%$. Llama la atención este hallazgo en la serie de adultos ya que está descrito que los tumores secundarios o metastásicos son entre 20 a 50 veces más frecuentes que los primarios ${ }^{2,34,36}$. No tenemos una explicación clara para este hecho, salvo que muchos de estos tumores no son diagnosticados, por corresponder a metástasis en una patología terminal no operable. En el caso de los niños coincidimos con lo descrito, en que el tumor primario es el más frecuente ${ }^{3,4,7}$. En ambas series, los tumores primarios fueron benignos: en niños 10 de 11 y en adultos 18 de 19, confirmando en este caso lo descrito en la literatura ${ }^{7-9}$.

El tumor primario más frecuente en niños es el rabdomioma y en los adultos el mixoma ${ }^{15,23}$, lo que concuerda con nuestros datos; en niños predominaron rabdomiomas (54\%) y en adultos mixomas (56\%), diferencia que fue estadísticamente significativa. En adultos no hubo casos de rabdomioma, asociación poco frecuente $\mathrm{y}$ en niños hubo un caso con mixoma, lo que también es infrecuente ${ }^{28,29}$.

Frente a una masa intracavitaria en niños, en primer lugar debe sospecharse un rabdomioma, especialmente si es de ubicación ventricular (Figura 1). Son benignos y regresan espontáneamente en al menos $54 \%$ de los casos, sin embargo, pueden tener indicación quirúrgica si presentan manifestaciones clínicas como compromiso hemodinámico o disrritmia refractaria, síntomas que son más frecuentes en edades tempranas ${ }^{10,11,17,22}$. Se pueden observar desde la época fetal y en este caso suelen ser múltiples en más de $90 \%$ de los $\operatorname{casos}^{4,18}$. Se asocian a esclerosis tuberosa en al menos $50 \%$, patología que se puede transmitir de forma autosómica dominante y se caracteriza por el desarrollo de tumores llamados hamartomas que afectan cerebro, piel, riñones, esqueleto,

Tabla 3. Comparación serie infantil y adultos

\begin{tabular}{|c|c|c|c|c|c|}
\hline & \multicolumn{2}{|c|}{ Niños } & \multicolumn{2}{|c|}{ Adultos } & $\mathrm{P}$ \\
\hline Tumores prim. benignos & $10 / 13$ & $77 \%$ & $18 / 25$ & $72 \%$ & NS \\
\hline Rabdomioma & $7 / 13$ & $54 \%$ & $0 / 25$ & $0 \%$ & $\mathrm{~S}$ \\
\hline Mixoma & $1 / 13$ & $8 \%$ & $14 / 25$ & $56 \%$ & $\mathrm{~S}$ \\
\hline Fibroma & $1 / 13$ & $8 \%$ & $0 / 25$ & $0 \%$ & $\mathrm{~S}$ \\
\hline Seudotumor & $1 / 13$ & $8 \%$ & $0 / 25$ & $0 \%$ & S \\
\hline Fibroelastoma & $0 / 13$ & $0 \%$ & $3 / 25$ & $12 \%$ & S \\
\hline Hamartoma & $0 / 13$ & $0 \%$ & $1 / 25$ & $4 \%$ & S \\
\hline Tumores prim. malignos & $1 / 13$ & $8 \%$ & $1 / 25$ & $4 \%$ & NS \\
\hline Tumores metastásicos & $2 / 13$ & $15 \%$ & $6 / 25$ & $24 \%$ & NS \\
\hline Sintomáticos & $10 / 13$ & $77 \%$ & $23 / 25$ & $92 \%$ & NS \\
\hline Ubicación auricular & $6 / 13$ & $46 \%$ & $18 / 25$ & $72 \%$ & S \\
\hline Ubicación ventricular & $7 / 13$ & $54 \%$ & $4 / 25$ & $16 \%$ & S \\
\hline Cirugía & $4 / 13$ & $31 \%$ & $21 / 25$ & $84 \%$ & S \\
\hline Vivos & $9 / 13$ & $69 \%$ & $20 / 25$ & $80 \%$ & NS \\
\hline
\end{tabular}

S: P <0,05; NS: no significativo. 


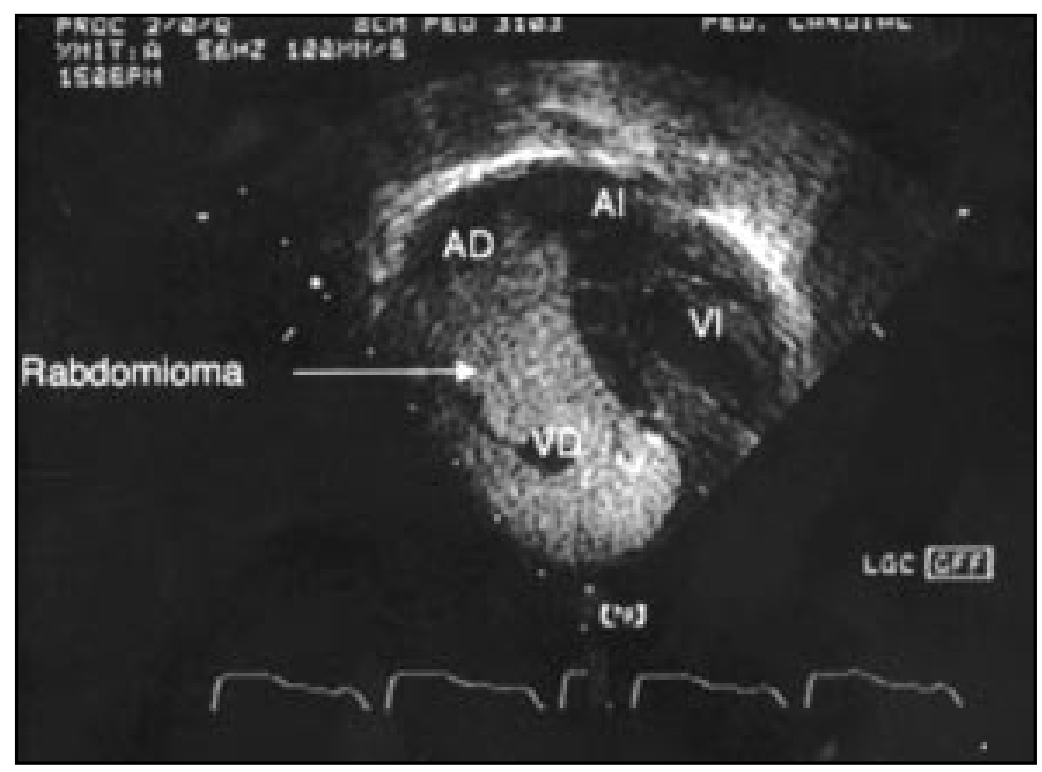

Figura 1. Ecocardiograma transtorácico, visión de cuatro cámaras, de un lactante con un rabdomioma múltiple en ventrículo y aurícula derecha.

$\mathrm{AD}$ : aunícula derecha, VD: ventrículo derecho, AI: aurícula izquierda, VI: ventrículo izquierdo, flecha indicando el tumor.

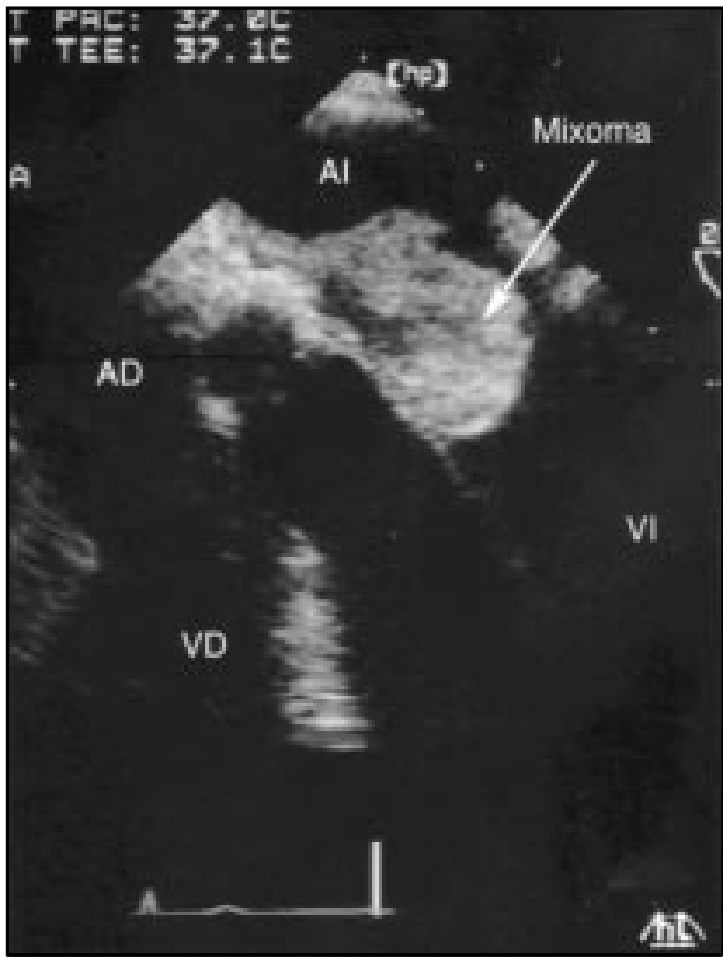

Figura 2. Ecocardiograma transesofágico, visión de cinco cámaras, de un adulto con un mixoma de aurícula izquierda. AD: aurícula derecha, VD: ventrículo derecho, AI: aurícula izquierda, VI: ventrículo izquierdo, flecha indicando el tumor. retina y corazón, el que está comprometido en 30$50 \%$ de los casos ${ }^{19-22}$. En esta serie hubo 3 diagnósticos fetales y en dos de ellos los tumores eran múltiples. El segundo tumor más frecuente en la infancia es el fibroma, generalmente solitario, de ubicación ventricular, sin regresión espontánea y usualmente requieren tratamiento quirúngico ${ }^{32}$. En nuestra serie hubo un caso (8\%).

El mixoma es el tumor más frecuente en adultos y corresponde a $50 \%$ de los tumores benignos. Son únicos y se observan frecuentemente de la tercera a sexta década de la vida, predominando en mujeres ${ }^{23-25}$. En nuestra serie correspondió a $56 \%$ de los casos (Figura 2). La presencia de mixomas múltiples se asocia al Complejo Carney, síndrome autosómico dominante y que suele presentarse en pacientes jóvenes. Además, presentan otras neoplasias, hiperfunción endocrina y una variedad de lesiones cutáneas hiperpigmentadas ${ }^{30}$.

Los tumores malignos primarios son bastante raros, aunque en algunas series alcanza a 10\%. La información sobre su tratamiento y pronóstico se obtiene de casos aislados y series pequeñas ${ }^{2,3}$. El tipo más frecuente es el sarcoma ${ }^{33}$. En los niños de esta serie hubo un caso de rabdomiosarcoma y en adultos un leiomiosarcoma, con 8\% y 4\%, respectivamente.

Los tumores malignos secundarios o metástasis cardiacas tienen tipo y comportamiento distinto 
según la edad. En niños se describen neuroblastomas y en adultos carcinomas y melanomas. La incidencia de linfomas cardíacos ha ido aumentando en el último tiempo, asociado al síndrome de inmunodeficiencia adquirida ${ }^{34-36}$. En esta serie se observaron 2 niños y seis adultos con tumores malignos secundarios, con $15 \%$ y $24 \%$, respectivamente. Cabe destacar que sólo uno de dos en niños y dos de 6 en adultos fueron certificados como tumor metastásico, ya que el resto fueron declarados inoperables y no podemos descartar que se tratara de un trombo intracardíaco secundario al síndrome de hipercoagulabilidad que acompaña a estas patologías.

La localización depende del tipo de tumor. Los niños de esta serie tenían preferentemente localización ventricular, ya que el tumor más frecuente en ellos es el rabdomioma y en los adultos fue auricular por ser el mixoma en más frecuente. Pueden además localizarse en el endocardio, miocardio o epicardio y dependiendo de su localización, tamaño y número pueden causar síntomas e incluso muerte ${ }^{27,29}$.

Los síntomas descritos en la serie infantil y adulta fueron similares a aquellos descritos en la literatura. En la serie infantil, 23\% fueron asintomáticos y detectados ecocardiográficamente versus $8 \%$ de asintomáticos en adultos, diferencia no significativa. Dentro de los síntomas relevantes están las amitmias: bloqueo aurículo-ventricular, taquicardias ventriculares y supraventriculares como el síndrome de Wolf Parkinson White, flutter y fibrilación auricular ${ }^{22,37-40 .}$ En algunos rabdomiomas asociados a esclerosis tuberosa se han observado amitmias refractarias a tratamiento, las que pueden ser tratadas con ablación por radiofrecuencia ${ }^{22,42}$. En nuestra senie de niños hubo dos casos de taquicardia paroxística supraventricular, falleciendo uno de ellos por insuficiencia cardíaca y tres adultos con fibrilación auricular. En todos ellos sólo se realizó tratamiento farmacológico. En cuanto a los pacientes con mixomas, ellos se manifiestan por alguno de los síntomas de la triada: embolia, obstrucción intracardíaca y síntomas sistémicos como fatiga, fiebre, rash enitematoso, artralgias, mialgias y pérdida de peso $23,24,28,41$. Esto explicańa la mayor frecuencia de síntomas en la senie de adultos, donde predominan los mixomas.

Los tumores cardíacos pueden coexistir con cardiopatías congénitas. Se ha descrito la asociación de rabdomioma con anomalía de Ebstein e hipoplasia de la válvula tricúspide, mixomas múltiples con doble salida de ventrículo derecho y fibroma con atresia pulmonar ${ }^{9,24}$. En esta revisión encontramos sólo un niño con una comunicación interventricular asociada y un paciente adulto con una comunicación interauricular.

En la mayoría de los tumores cardíacos de los niños el manejo es médico, ya que se trata de rabdomiomas y la cirugía está indicada en casos de tumores sintomáticos y también en tumores malignos, mejorando el pronóstico de estos últimos ${ }^{12-}$ 14,32 . En los adultos, la conducta quirúrgica agresiva ha tenido buenos resultados ${ }^{5,26}$. En esta serie, cuatro pacientes de la serie infantil (31\%) y 21 pacientes adultos (84\%) se sometieron a cirugía, diferencia que es estadísticamente significativa.

Antes del estudio por imágenes, los diagnósticos se realizaban por autopsias. Actualmente es la ecocardiografía la modalidad diagnóstica de elección, ya que es no invasiva, permite el diagnóstico en periodo antenatal y en pacientes asintomáticos, entrega información respecto a la localización, características del tejido, significación hemodinámica y derrame pericárdico asociado $6,16,25$. Su limitación está dada por la ventana del paciente, la que es peor en adultos. La ecotransesofágica es invasiva, pero mejora las imágenes y permite investigar mejor el apéndice de la aurícula izquierda, la vena cava superior y la superficie anterior del corazón ${ }^{43}$. En esta serie el método diagnóstico fundamental fue la ecocardiografía en sus tres modalidades, transtorácica, transesofágica y fetal. La ecocardiografía 3D está desarrollándose como un método útil en el diagnóstico de estas patologías ${ }^{44}$. Del grupo estudiado, sólo en un caso de la serie infantil se utilizó la resonancia como método diagnóstico complementario. Esta técnica, al igual que la tomografía axial computarizada proporciona información adicional ${ }^{45,46}$. La angiografía sólo se utilizó en un paciente de la serie de adultos, método que puede ser de utilidad para evaluar compromiso de arterias coronarias en grandes tumores, pero que cada vez se realiza menos.

En los últimos años han ido aumentando los reportes de pacientes con tumores cardíacos fetales, detectados en la evaluación ecográfica antenatal $^{47}$. En esta serie describimos 3 casos (Figura 3). Frente a este hallazgo, se ha sugerido la posibilidad de encontrar mayor incidencia de esclerosis tuberosa ${ }^{48}$ y por ello es de utilidad complementar 


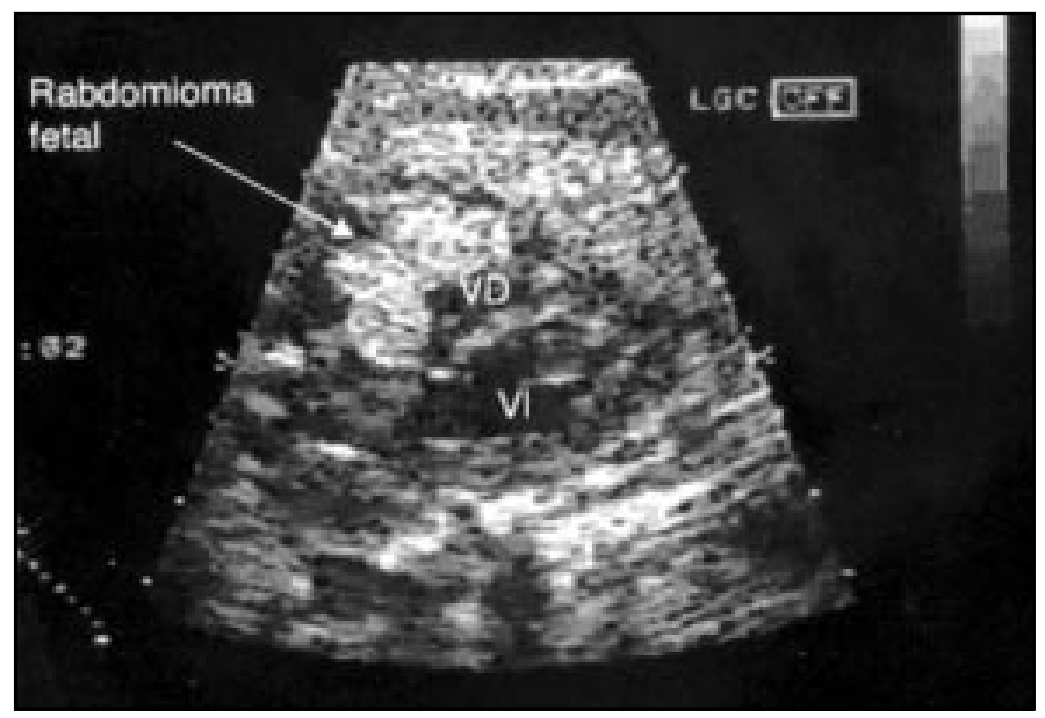

Figura 3. Ecocardiograma fetal, visión de cuatro cámaras, de un feto con un rabdomioma múltiple de ventrículo derecho.

AD: aurícula derecha, VD: ventrículo derecho, AI: aurícula izquierda, VI: ventrículo izquierdo, flecha indicando el tumor.

el estudio con resonancia magnética para investigar lesiones en otros órganos ${ }^{49}$.

Durante el seguimiento de los niños se espera que los rabdomiomas tengan una regresión espontánea y por ello deben tener una evaluación periódica con ecocardiografía, que la certifique y que además permita detectar complicaciones. En el caso de los adultos, el seguimiento con ecocardiográfía de los mixomas operados puede detectar recidivas por una resección insuficiente del tumor.

\section{REFERENCIAS}

1. Lam KY, Dickens P, Chan AC. Tumors of the heart. A 20-year experience with a review of 12,485 consecutive autopsies. Arch Pathol Lab Med 1993; 117: 1027-31.

2. Shapiro LM. Cardiac tumours: diagnosis and management. Heart 2001; 85: 218-22.

3. Freedom RM, Lee KJ, MacDonald C, Taylor G. Selected aspects of cardiac tumors in infancy and childhood. Pediatr Cardiol 2000; 21: 299-316.

4. IsAacs $\mathrm{H} \mathrm{JR}$. Fetal and neonatal cardiac tumors. Pediatr Cardiol 2004; 25: 252-73.

5. Miralies A, Bracamonte L, Soncul H, Díaz del CASTILO R, AKHTAR R, Bors V et AL. Cardiac tumors:
En conclusión, es una patología relevante y poco frecuente y que se presenta y se trata en forma diferente en niños y adultos, siendo el rabdomioma, un tumor exclusivo de los niños el más frecuente en ellos, y el mixoma el tumor más frecuente de los adultos. Ambos tumores primarios y benignos. Los tumores malignos primarios y metastáticos se presentaron con baja frecuencia en ambos grupos.

Finalmente, por tratarse de una patología poco frecuente requiere de estudios colaborativos multicéntricos, dado que sólo así podremos conocer mejor su comportamiento.

clinical experience and surgical results in 74 patients. Ann Thorac Surg 1991; 52: 886-95.

6. Aluzad A, Seward JB. Echocardiographic features of genetic diseases: part 5. Tumors. J Am Soc Echocardiogr 2000; 13: 421-6.

7. Reynen K. Frequency of primary tumors of the heart. Am J Cardiol 1996; 77: 107.

8. Beghetti M, Gow RM, Haney I, Mawson J, Wimams WG, FREEDOM RM. Pediatric primary benign cardiac tumors: a 15-year review. Am Heart J 1997; 134: 1107-14.

9. BECKER AE. Primary heart tumors in pediatric age group: a review of salient pathologic features relevant for clinicians. Pediatr Cardiol 2000; 21: $317-23$. 
10. Hwa J, Ward C, Nunn G, Cooper S, Lau KC, Sholefr G. Primary intraventricular cardiac tumors in children: contemporary diagnostic and management options. Pediatr Cardiol 1994; 15: 233-7.

11. Ohara Y, Hiasa Y, Hosokawa S, Yamaguchi $K$, Ogura R, Ogata T et al. Primary cardiac tumor presenting as obstruction of the left ventricular outflow tract. Circ J 2004; 68: 961-3.

12. Stiler B, Hetzer R, Meyer R, Dittrich S, Pees C, AifXI-Meskishvil V et al. Primary cardiac tumours: when is surgery necessary? Eur J Cardiothorac Surg 2001; 20: 1002-6.

13. Takach TJ, Reul GJ, Ott DA, Cooley DA. Primary cardiac tumors in infants and children: immediate and long-term operative results. Ann Thorac Surg 1996; 62: 559-64.

14. Tazelaar HD, Locke TJ, McGregor CG. Pathology of surgically excised primary cardiac tumors. Mayo Clin Proc 1992; 67: 957-65.

15. Smythe JF, Dyck JD, Smalhorn JF, Freedom RM. Natural history of cardiac rhabdomyoma in infancy and childhood. Am J Cardiol 1990; 66: 1247-9.

16. Di Luang C, Ko SF, Huang SC. Echocardiographic evaluation of cardiac rhabdomyoma in infants and children. J Clin Ultrasound 2000; 28: 381-6.

17. Black MD, Kadletz M, SMaLhorn JF, Freedom RM. Cardiac rhabdomyomas and obstructive left heart disease: histologically but not functionally benign. Ann Thorac Surg 1998; 65: 1388-90.

18. Paladini D, Palmieri S, Russo MG, Pacileo G. Cardiac multiple rhabdomyomatosis: prenatal diagnosis and natural history. Ultrasound Obstet Gynecol 1996; 7: 84-5.

19. Webi DW, Thomas RD, Osborne JP. Cardiac rhabdomyomas and their association with tuberous sclerosis. Arch Dis Child 1993; 68: 367-70.

20. DiMario FJ Jr, Diana D, Leopold H, Chameides L. Evolution of cardiac rhabdomyoma in tuberous sclerosis complex. Clin Pediatr (Phila) 1996; 35: 615-9.

21. Matsumura M, Nishioka K, Yamashita K, YoshibayasHi M, ОкUno T, Konishi J ET aL. Evaluation of cardiac tumors in tuberous sclerosis by magnetic resonance imaging. Am J Cardiol 1991; 68: 281-3.

22. Mas C, Penny DJ, Menahem S. Pre-excitation syndrome secondary to cardiac habdomyomas in tuberous sclemosis. J Paediatr Child Health 2000; 36: 84-6.

23. Reynen K. Cardiac myxomas. N Engl J Med 1995; 333: 1610-7.
24. Pinede L, Duhaut P, Loire R. Clinical presentation of left atrial cardiac myxoma. A series of 112 consecutive cases. Medicine (Baltimore) 2001; 80: 159-72.

25. Grebenc ML, Rosado-de-Christenson ML, Green CE, BurKe AP, Galvin JR. Cardiac myxoma: imaging features in 83 patients. Radiographics 2002; 22: 673-89.

26. Bortolotti U, Maraglno G, Rubino M, Santini F, Mazzucco A, Milano A et al. Surgical excision of intracardiac myxomas: a 20-year follow-up. Ann Thorac Surg 1990; 49: 449-53.

27. Vassiladis N, Vassiladis K, Karkaveias G. Sudden death due to cardiac myxoma. Med Sci Law 1997; 37: 76-8.

28. Tonz M, Laske A, Carrel T, da S, V, Real F, Turina M. Convulsions, hemiparesis and central retinal artery occlusion due to left atrial myxoma in child. Eur J Pediatr 1992; 151: 652-4.

29. Cimers AM, van UH, Lala S, Vanderdonck KH, HaRTMAn E. Massive biatrial myxomas in a child. Pediatr Cardiol 1999; 20: 150-1.

30. Carney JA. The Carney complex (myxomas, spotty pigmentation, endocrine overactivity, and schwannomas). Dermatol Clin 1995; 13: 19-26.

31. Das Narla L, Siddigi NH, Hinsbergen EA. Inflammatory pseudotumor of the right atrium. Pediatr Radiol 2001; 31: 351-3.

32. Burke AP, Rosado-de-Christenson M, Templeton PA, VIRMANI R, BURKE AP. Cardiac fibroma: clinicopathologic correlates and surgical tratment. J Thorac Cardiovasc Surg 1994; 108: 862-70.

33. Putnam JB Jr, Sweeney MS, Colon R, Lanza LA, Frazier OH, Cooley DA. Primary cardiac sarcomas. Ann Thorac Surg 1991; 51: 906-10.

34. Hun J, Noн CI, Kim YW, Chог JY, Yun YS, Shin HY ET AL. Secondary cardiac tumor in children. Pediatr Cardiol 1999; 20: 400-3.

35. Balasubramanyam A, Waxman M, Kazal HL, Lee MH. Malignant lymphoma of the heart in acquired immune deficiency syndrome. Chest 1986; 90: 243-6.

36. Martínez-Guerra G, Ruano-Aguilar J, Rivera-Luna R, Cárdenas-Cardos R, Avila-Ramírez E, Braun-Roth G ET AL. Wilms tumor with intracardiac extension. Eur J Pediatr Surg 1992; 2: 56-9.

37. Cere E, Lombardi A, Vinew S, Di PG. Concomitant left atrial myxoma and atrial fibrillation in patients with peripheral embolism. Ital HeartJ Suppl 2002; 3: 232-4. 
38. Muhler EG, Kienast W, TuRNISKI-Harder V, von BERNUTH G. Arrythmias in infants and children with primary cardiac tumours. Eur Heart J 1994; 15: $915-21$.

39. O’Callaghan FJ, Clarke AC, Jofre H, Keeton B, Martin R, Salmon A et al. Tuberous sclerosis complex an Wolff Parkinson White syndrome. Arch Dis Child 1998; 78: 159-2.

40. Cha'ban FK, Cohen-Overbeer TE, Frohn-Mudder IM, WLADIMIROFF JW. Multiple intracardiac tumors: spontaneus prenatal recovery of fetal bradyarrhythmia. Ultrasound Obstet Gynecol 1996; 8: 120-2.

41. Bayir H, Morem PJ, Smith TH, Biancanielo TA. A left atrial myxoma presenting as a cerebrovascular accident. Pediatr Neurol 1999; 21: 569-72.

42. Kohl V, Mangru N, Pearse LA, Cantwell P, Young ML. Radiofrequency ablation of ventricular tachycardia in an infant with cardiac tumors. Am Heart J 1996; 132: 198-200.

43. ReEder GS, Khandheria BK, Seward JB, TajiK AJ. Transesophageal echocardiography and cardiac masses. Mayo Clin Proc 1991; 66: 1101-9.

44. Espínola-Zavaleta N, Morales GH, Vargas-Barron J, Keirns C, Fraustro AA. Three-dimensional transe- sophageal echocardiography in tumors of the heart. J Am Soc Echocardiogr 2002; 15: 972-9.

45. Araoz PA, Mulvagh SL, Tazelaar HD, Julsrud PR, BREEN JF. CT and MR imagin of bening primary cardiac neoplasm with echocardiographic correlation. Radiographic 2000; 20: 1303-19.

46. Gulati G, Sharma S, Kothari SS, Juneja R, Saxena A, TALWAR KK. Comparison of echo and MRI in the imaging evaluation of intracardiac masses. Cardiovasc Intervent Radiol 2004; 27: 459-69.

47. Houey DG, Martin GR, Brenner JI, Fyfe DA, Huhta JC, Kieinman CS ET aL. Diagnosis and management of fetal cardiac tumors: a multicenter experience and review of published reports. J Am Coll Cardiol 1995; 26: 516-20.

48. Journel $H$, Roussey M, Piais MH, Milon J, Almange C, Le Marec B. Prenatal diagnosis of familiar tuberous sclerosis following detection of cardiac rhabdomyoma by ultrasound. Prenatal Diagn 1986; 6: 283-9.

49. Sonigo P, Elmaleh A, Fermont L, Delezoide AL, Mirlesse V, Brunele F. Prenatal MRI diagnosis of fetal cerebral tuberous sclerosis. Pediatr Radiol 1996; 26: 1-4. 